\title{
Caractérisation physico-chimique des eaux d'alimentation de la ville de Tijikja (Mauritanie)
}

\author{
Mohamed EL Kory OULD CHEIKH ${ }^{1 *}$, Kacem EL KACEMI ${ }^{2}$ et Larbi IDRISSI $^{3}$ \\ ${ }^{1}$ Laboratoire de Contrôle de la Qualité des Eaux, Institut National de Recherches en Santé Publique, BP 695 \\ Nouakchott (Mauritanie). \\ ${ }^{2}$ Laboratoire d'Electrochimie et de Chimie Analytique, Faculté des Sciences de Rabat (Maroc). \\ ${ }^{3}$ Département de Toxicologie, d'Hydrologie et d'Hygiène Environnementale, Institut National d'Hygiène- \\ Rabat (Maroc). \\ *Auteur correspondant, E-mail : mouhamed_elkory@yahoo.fr, Tél. : 0022236344781.
}

\section{RESUME}

Les ressources en eau de la Mauritanie sont limitées alors que ses besoins en eau augmentent. Dans le but d'étudier les caractéristiques physico-chimiques de la qualité des eaux d'alimentation de la ville de Tijikja capitale de la Wilaya du Tagant (région au centre de la Mauritanie) des analyses physicochimiques ont été effectués au niveau des quatre forages qui alimentent la ville ainsi qu'au niveau d'un robinet, d'une potence pour les citernes et de trois puits avec des paramètres physico-chimiques tels que la température, le $\mathrm{pH}$, la conductivité électrique, le TDS, la dureté, les ions calcium, magnésium, sodium, potassium, les chlorures, les bicarbonates, les nitrites, les nitrates, l'ammonium, les sulfates, le fluorure et les métaux lourds (fer, zinc, chrome, cadmium, plomb et le cuivre). Les résultats des analyses physico-chimiques montrent que le $\mathrm{pH}$ est proche de la neutralité, il varie entre 7,1 et 7,6; la conductivité est élevée au niveau du forage d'El Arghoub $(1885 \mu \mathrm{S} / \mathrm{Cm})$. Elle est moyenne au niveau des puits. On remarque une moyenne minéralisation variant entre 250,5 et $352 \mathrm{mg} / \mathrm{L}$ due à une conductivité moyenne au niveau des autres forages. Les chlorures et le potassium dépassent les normes de l'OMS pour le forage T1 et le puits P3, ils sont respectivement $310 \mathrm{mg} / \mathrm{L} \mathrm{et} 32 \mathrm{mg} / \mathrm{L}$. Les concentrations des ions calcium, magnésium, sodium, sulfate et fluorures sont toujours inférieures aux normes de l'OMS de l'eau potable pour l'ensemble des échantillons prélevés. En ce qui concerne les composés azotés on observe des concentrations négligeables en nitrites et que l'ammonium n'a pas été détecté pour tous les échantillons des eaux d'alimentation de la ville de Tijikja. Cependant les teneurs en nitrates sont inférieures à $5 \mathrm{mg} / \mathrm{L}$ pour les échantillons prélevés sauf pour les échantillons prélevés au niveau du puits P1, P3 et au forage $\mathrm{T} 1$ avec des teneurs respectives de $36,7 \mathrm{mg} / \mathrm{L}, 43,5 \mathrm{mg} / \mathrm{L}$ et $36,44 \mathrm{mg} / \mathrm{L}$. Les eaux des forages de Tijikja analysées montrent l'existence de métaux lourds tels que le chrome, le zinc, le cadmium et le plomb à des concentrations variables restant toujours inférieures aux normes de l'OMS. Dans l'espoir que la Mauritanie, à l'instar de ses voisins maghrébins et ouest africains mettra en place des normes en matière de l'eau potable. Dans ce cas la caractérisation physico-chimique des eaux d'alimentation de cette ville importante qui est Tijikja va servir comme base de données.

(C) 2011 International Formulae Group. All rights reserved.

Mots clés : Physico-chimique, eau Tijikja, puits, forages, Mauritanie.

\section{INTRODUCTION}

La Mauritanie est un pays déficitaire en eau et ce problème est particulièrement important à cause du climat. En effet la faible pluviométrie, la nature géologique du pays et l'importance de l'évaporation font que le pays 
est pauvre en ressources hydriques. Les ressources en eau de la Mauritanie sont limitées alors que ses besoins en eau augmentent.

Les sources et les eaux souterraines sont traditionnellement les ressources en eau privilégiées pour l'eau potable, car plus à l'abri des pollutions que les eaux de surface. Toutefois, certaines caractéristiques minimales sont exigées pour qu'elles puissent servir à la production d'eau potable qui doitelle- même satisfaire à des normes de qualité physico-chimique et biologique (WHO, 1996).

Des études similaires ont été effectuées au niveau des pays voisins pour la caractérisation des eaux souterraines (Bricha et al, 2007 ; Derwich et al., 2010 ; Guergazi et Achour, 2005). En général les problèmes les plus rencontrés dans ces pays sont toujours ceux de la salinité, de la présence des teneurs élevées en nitrates,...

La ville de Tijikja est alimentée en eau de consommation à partir de 4 forages dans la nappe alluviale ou faille du champ captant d'oued Tijikja et dans la nappe des calcaires du champ captant le forage d'El Argoub (T1) qui refoulent leurs eaux vers un réservoir. A cela s'ajoute l'injection directe de plusieurs eaux des forages (TKF1, TKF2 et FATTM) dans le réseau de distribution.

L'objectif de notre étude est de faire une caractérisation physico-chimique des eaux d'alimentation de la ville de Tijikja en effectuant des prélèvements au niveau des 4 forages, 3 puits, un robinet et une potence avec des paramètres physico-chimiques.

\section{MAERIEL ET METHODES Milieu d'étude}

La ville de Tijikja capitale de la Wilaya du Tagant (Mauritanie) est située sur un aquifère discontinu. L'analyse de la coupe géologique montre que les ressources mobilisables en eau sont contenues dans les 3 types d'aquifères caractéristiques du Tagant :

- l'aquifère discontinu des grès ;

- l'aquifère discontinu des calcaires ;

- l'aquifère continu.
Avant 2002, la ville comptait 30 bornes fontaines. Depuis, un réseau a été installé et aujourd'hui on dénombre 4 bornes fontaines et 4 forages autour de la ville. Le principal problème est la destruction des ouvrages pendant l'hivernage (période de grosses pluies). D'où des restrictions, des coupures d'eau et des baisses de pression. Dans cette région désertique la Wilaya du Tagant n'a bénéficié que de faible pluies (Centre Régional Agrhymet, 2005) la seule activité agricole rentable est le palmier. De grandes palmeraies permettent une récolte de datte pendant la «guetna » (de juillet à septembre). Cette activité est très consommatrice d'eau. Les paysans utilisent un système traditionnel, le puits Chadouf, puits à balancier permettant grâce à une longue perche de réduire les efforts humains lors de la remontée du sot. Ces puits puisent une eau située à faible profondeur environ 10 à 15 mètres.

\section{Prélèvements et analyses}

Les points de prélèvement des échantillons d'eaux au niveau de la ville de Tijikja sont :

- Les quatre forages qui alimentent la ville (T1, TKF1, TKF2 et FATTM).

- Deux points du réseau d'alimentation (Robinet du consommateur (R) et potence (PT) pour l'alimentation des citernes).

- $\quad$ Trois puits (P1, P2 et P3).

Les paramètres physico-chimiques étudiés sont la température, le $\mathrm{pH}$, les Solides totaux dissous (TDS), la conductivité électrique, la dureté totale (TH), le calcium, le magnésium, le sodium, le potassium, les bicarbonates, les chlorures, les sulfates, les nitrates, les nitrites, ammonium, Les fluorures, le fer $(\mathrm{Fe})$, le chrome $(\mathrm{Cr})$, le cuivre $(\mathrm{Cu})$, le zinc $(\mathrm{Zn})$, le cadmium $(\mathrm{Cd})$ et le plomb $(\mathrm{Pb})$.

Le $\mathrm{pH}$ et la température ont été mesuré par un pH-mètre muni d'une sonde de température de marque HANNA de type HI 8314. La conductivité est mesurée grâce à un conductimètre de marque HANNA de type HI8733. Le titre hydrotimétrique $\mathrm{TH}$, le calcium et le magnésium sont déterminés par 
complexométrie à l'EDTA et les bicarbonates ont été analysés par méthode volumétrique à l'acide chlorhydrique (Rodier, 1996). Le sodium et le potassium sont déterminés par spectromètre à flamme de marque Corning type 410. Les chlorures sont déterminés par la méthode de Mohr. Les sulfates, les nitrates, l'ammonium et les nitrites sont déterminés par colorimétrie sur un spectrophotomètre UVVisible de type 722 S Beijing. Le fluor est déterminé par potentiométrie en utilisant une électrode spécifique PF-1 (Shanghai Weiye Instrument Plant). Les métaux lourds (fer, chrome, zinc, cuivre, cadmium et plomb) sont analysés pour les échantillons des eaux des forages T1, TKF1, TKF2, FATTM, R et PT au laboratoire des polluants inorganiques $\mathrm{du}$ département de toxicologie d'hydrologie et d'hygiène environnementale (Institut National d'Hygiène de Rabat) par un spectromètre d'absorption atomique à four graphite (AA 240Z) de marque VARIAN type GTA 120 piloté par un logiciel (Spectra).

\section{RESULTATS}

Les résultats de l'analyse des caractéristiques physico-chimiques des eaux de Tijikja sont présentés dans le Tableau 1 pour les eaux des forages et dans le tableau 2 pour les eaux des puits.

La température de nos échantillons est comprise entre 25,6 et $32,2{ }^{\circ} \mathrm{C}$ (Tableau 1), elle est acceptable pour les zones arides et semi arides. Le pH est compris entre 7,1 et 7,6 (Tableau 1).

La conductivité très forte elle dépasse $1000 \mu \mathrm{S} / \mathrm{cm}$ pour les échantillons T1, R et P1 (Tableaux 1 et 2). La concentration des sulfates est plus élevée au niveau des échantillons des puits $(120,4 \mathrm{mg} / \mathrm{L}$ pour P1) que pour les autres points prélevés qui ont une concentration inferieure à $30 \mathrm{mg} / \mathrm{L}$. La dureté totale minimale est de $13,01^{\circ} \mathrm{f}$ pour l'échantillon TKF2 et la teneur maximale est de 53,04 ${ }^{\circ} \mathrm{f}$ pour l'échantillon du forage $\mathrm{T} 1$. La concentration minimale des ions calcium a été observée au niveau du puits P2 (24,78 $\mathrm{mg} / \mathrm{L}$ ) tandis que la teneur maximale a été enregistrée au niveau du forage T1 $(56,91$ $\mathrm{mg} / \mathrm{L})$. La teneur minimale en magnésium est de $13,62 \mathrm{mg} / \mathrm{L}$ pour l'échantillon du forage TKF2 et la teneur maximale est de 94,36 $\mathrm{mg} / \mathrm{L}$ pour l'échantillon T1. La concentration du sodium enregistré varie entre $41 \mathrm{mg} / \mathrm{L}$ pour le puits $\mathrm{P} 2$ et $108 \mathrm{mg} / \mathrm{L}$ pour le forage $\mathrm{T} 1 \mathrm{de}$ même, le potassium varie entre $3 \mathrm{mg} / \mathrm{L}$ pour l'échantillon du forage TKF2 et $32 \mathrm{mg} / \mathrm{L}$ pour le puits P3. Les bicarbonates varient entre 136,64 mg/L pour l'échantillon du forage TKF1 et $341,6 \mathrm{mg} / \mathrm{L}$ pour le puits P1. La concentration des nitrates dans les eaux analysées du Tijikja est de 1,97 mg/L comme étant minimale pour l'échantillon de la potence PT et $43,5 \mathrm{mg} / \mathrm{L}$ comme étant maximale pour le puits $\mathrm{P} 3$. Les nitrites varient entre $0,004 \mathrm{mg} / \mathrm{L}$ pour le forage $\mathrm{TKF} 1$ et $0,0146 \mathrm{mg} / \mathrm{L}$ pour le FATTM. L'ammonium n'a pas été détecté au niveau des échantillons d'eau souterraine de Tijikja analysées.

En ce que concerne le fluor dans les eaux de Tijikja il varie entre une concentration de $0,81 \mathrm{mg} / \mathrm{L}$ pour $\mathrm{T} 1$ et $0,42 \mathrm{mg} / \mathrm{L}$ pour les forages TKF1 et FATTM. On remarque que les puits ont une concentration en fluorure la plus élevé $1,38 \mathrm{mg} / \mathrm{L}$ pour $\mathrm{P} 1$ est environ 0,60 $\mathrm{mg} / \mathrm{L}$ pour $\mathrm{P} 2$ et $\mathrm{P} 3$ en ions fluorures (Tableau 2). Nous observons une forte concentration en chlorures dépassant les normes de l'OMS pour le forage $\mathrm{T} 1$, les autres échantillons ont une concentration en chlorures moins de 100 $\mathrm{mg} / \mathrm{L}$ sauf pour les échantillons $\mathrm{R}, \mathrm{P} 1$ et $\mathrm{P} 3$ respectivement $251 \mathrm{mg} / \mathrm{L}, 107,21 \mathrm{mg} / \mathrm{L}$ et 190,52 mg/L.

L'analyse des métaux lourds des forages T1, TKF1, TKF2, FATTM, R et PT de Tijikja montre l'absence du fer et du cuivre. Le chrome varie entre $1,05 \mu \mathrm{g} / \mathrm{L}$ pour l'échantillon de la potence PT et 1,72 $\mu \mathrm{g} / \mathrm{L}$ pour l'échantillon du forage T1. Le zinc varie entre 42,5 $\mu \mathrm{g} / \mathrm{L}$ pour l'échantillon de la potence PT et $51,1 \mu \mathrm{g} / \mathrm{L}$ pour le forage TKF2. La concentration du cadmium varie entre $0,002 \mu \mathrm{g} / \mathrm{L}$ pour le forage FATTM et 0,019 $\mu \mathrm{g} / \mathrm{L}$ pour le forage TKF1. Le plomb dans les eaux analysées montre une concentration qui varie entre $0,396 \mu \mathrm{g} / \mathrm{L}$ pour l'échantillon de la potence PT et $1,448 \mu \mathrm{g} / \mathrm{L}$ pour l'échantillon du forage $\mathrm{T} 1$. 
Tableau 1 : Caractéristiques physico-chimiques des échantillons d'eaux de Tijikja.

\begin{tabular}{|c|c|c|c|c|c|c|}
\hline Paramètres & F (T1) & F (TKF1) & F(TKF2) & F(ATTM) & $\mathbf{R}$ & PT \\
\hline $\mathbf{T}^{\circ} \mathbf{C}$ & 25,8 & 25,8 & 25,8 & 25,7 & 26,6 & 25,6 \\
\hline PH & 7,19 & 7,20 & 7,60 & 7,20 & 7,33 & 7,55 \\
\hline S.T.D. mg/L & 942,5 & 292,0 & 352,0 & 250,5 & 788,5 & 305,5 \\
\hline Conductivité $\mu \mathrm{S} / \mathrm{Cm}$ & 1885,00 & 584,00 & 704,00 & 501,00 & 1577,00 & 611,00 \\
\hline TH ${ }^{\circ} \mathbf{f}$ & 53,04 & 17,21 & 13,01 & 15,01 & 43,64 & 15,01 \\
\hline $\mathrm{Ca}^{2+} \mathrm{mg} / \mathrm{L}$ & 56,91 & 37,28 & 29,66 & 32,06 & 47,29 & 34,47 \\
\hline $\mathrm{Mg}^{2+} \mathrm{mg} / \mathrm{L}$ & 94,36 & 17,99 & 13,62 & 17,02 & 77,34 & 15,56 \\
\hline $\mathrm{Na}^{+} \mathrm{mg} / \mathrm{L}$ & 108,00 & 52,00 & 74,00 & 49,00 & 98,00 & 62,00 \\
\hline $\mathrm{K}^{+} \mathbf{m g} / \mathrm{L}$ & 9,00 & 5,00 & 3,00 & 4,00 & 8,00 & 4,00 \\
\hline $\mathrm{HCO}_{3}{ }^{-} \mathrm{mg} / \mathrm{L}$ & 169,8 & 136,64 & 187,88 & 168,48 & 161,04 & 180,56 \\
\hline $\mathrm{Cl}^{-} \mathrm{mg} / \mathrm{L}$ & 310,00 & 78,00 & 80,00 & 42,00 & 251,00 & 66,00 \\
\hline $\mathrm{SO}_{4}{ }^{2-} \mathrm{mg} / \mathrm{L}$ & 30,08 & 14,00 & 14,52 & 12,44 & 27,66 & 13,31 \\
\hline $\mathrm{NO}_{2}^{-} \mathrm{mg} / \mathrm{L}$ & 0,0065 & 0,0040 & 0,0065 & 0,0146 & 0,0057 & 0,0040 \\
\hline $\mathrm{NO}_{3}^{-} \mathrm{mg} / \mathrm{L}$ & 36,44 & 4,82 & 2,67 & 3,24 & 26,96 & 1,97 \\
\hline $\mathrm{NH}_{4}{ }^{+} \mathrm{mg} / \mathrm{L}$ & 0,00 & 0,00 & 0,00 & 0,00 & 0,00 & 0,00 \\
\hline$F^{-} \mathrm{mg} / \mathrm{L}$ & 0,81 & 0,42 & 0,52 & 0,42 & 0,67 & 0,45 \\
\hline $\mathrm{Fe}(\mathrm{mg} / \mathrm{L})$ & 0,00 & 0,00 & 0,00 & 0,00 & 0,00 & 0,01 \\
\hline $\operatorname{Cr}(\mu \mathrm{g} / \mathrm{L})$ & 1,72 & 1,66 & 1,10 & 1,06 & 1,35 & 1,05 \\
\hline $\operatorname{Zn}(\mu \mathrm{g} / \mathrm{L})$ & 47,7 & 45,9 & 51,1 & 43,1 & 46,2 & 42,4 \\
\hline $\mathrm{Cu}(\mu \mathrm{g} / \mathrm{L})$ & $<0,002$ & $<0,002$ & $<0,002$ & $<0,002$ & $<0,002$ & $<0,002$ \\
\hline $\operatorname{Cd}(\mu \mathrm{g} / \mathrm{L})$ & 0,003 & 0,019 & 0,003 & 0,002 & 0,011 & 0,003 \\
\hline $\mathrm{Pb}(\mu \mathrm{g} / \mathrm{L})$ & 1,448 & 0,451 & 0,415 & 0,435 & 1,211 & 0,396 \\
\hline
\end{tabular}

Tableau 2 : Caractéristiques physico-chimiques des échantillons d'eaux des puits de Tijikja.

\begin{tabular}{|c|c|c|c|}
\hline Paramètres & P1 & $\mathbf{P 2}$ & P3 \\
\hline $\mathbf{T}^{\circ} \mathbf{C}$ & 30,2 & 31,8 & 32,2 \\
\hline PH & 7,42 & 7,42 & 7,1 \\
\hline S.T.D. mg/L & 530,0 & 255,0 & 497,5 \\
\hline Conductivité $(\mu \mathrm{S} / \mathrm{Cm})$ & 1060 & 510 & 995 \\
\hline $\mathbf{T H}\left({ }^{\circ} \mathbf{f}\right)$ & 30,02 & 18,01 & 38,43 \\
\hline $\mathrm{Ca}^{2+}(\mathrm{mg} / \mathrm{L})$ & 25,65 & 31,26 & 54,51 \\
\hline $\mathrm{Mg}^{2+}(\mathrm{mg} / \mathrm{L})$ & 57,35 & 24,78 & 61,23 \\
\hline $\mathrm{Na}^{+}(\mathrm{mg} / \mathrm{L})$ & 92,00 & 41,00 & 105,00 \\
\hline $\mathrm{K}^{+}(\mathrm{mg} / \mathrm{L})$ & 12,00 & 5,00 & 32,00 \\
\hline $\mathrm{HCO}_{3}^{-}(\mathrm{mg} / \mathrm{L})$ & 341,60 & 207,40 & 190,8 \\
\hline $\mathrm{Cl}^{-}(\mathrm{mg} / \mathrm{L})$ & 107,21 & 40,82 & 190,52 \\
\hline $\mathrm{SO}_{4}{ }^{2-}(\mathrm{mg} / \mathrm{L})$ & 120,4 & 32,61 & 82,97 \\
\hline $\mathrm{NO}_{2}^{-}(\mathrm{mg} / \mathrm{L})$ & 0,00 & 0,00 & 0,00 \\
\hline $\mathrm{NO}_{3}^{-}(\mathrm{mg} / \mathrm{L})$ & 36,7 & 23,3 & 43,5 \\
\hline $\mathrm{NH}_{4}{ }^{+}(\mathrm{mg} / \mathrm{L})$ & 0,00 & 0,00 & 0,00 \\
\hline$F^{-}(\mathrm{mg} / \mathrm{L})$ & 1,38 & 0,59 & 0,63 \\
\hline
\end{tabular}




\section{DISCUSSION \\ Température et pH}

Il est primordial de connaître la température d'une eau. En effet elle joue un rôle très important dans la solubilité des sels et surtout des gaz. La température de nos échantillons est comprise entre 25,6 et $32,2^{\circ} \mathrm{C}$ (Tableau 1), elle est acceptable pour les zones arides et semi arides Selon les normes (OMS 2006), le $\mathrm{pH}$ d'une eau potable doit être compris entre 6.5 et 9.5 , le $\mathrm{pH}$ des eaux de Tijikja est donc très proche de la neutralité.

\section{Minéraux}

La dureté de l'eau (TH) montre que les eaux du forage $\mathrm{T} 1$ sont très dures avec un $\mathrm{TH}$ supérieurs à la norme de $50^{\circ} \mathrm{F}$, au niveau du robinet de consommateur (R) le $\mathrm{TH}$ est inferieur à cette norme $\left(43,64^{\circ} \mathrm{F}\right)$ du fait des injections des eaux douces des autres forages. Les puits de Tijikja ont des eaux moyennement dures à très dures (P3) (Memotec, 2005). La dissolution du $\mathrm{Ca}^{2+}$ et du $\mathrm{Mg}^{2+}$ résulte principalement de l'infiltration des eaux de surface à travers les formations rocheuses calcaires et dolomitiques. Cette dissolution est accrue par la présence dans l'eau de gaz carbonique provenant de l'atmosphère et des couches superficielles du sol (Tardat et Beaudry, 1984). La dureté possède des propriétés gênantes telles que formation de dépôts durs dans les canalisations du réseau (entartrage), gêne dans les opérations de lavage, etc...

\section{Salinité}

La conductivité électrique traduit le degré de minéralisation globale, elle nous renseigne sur le taux de salinité. La conductivité est très forte elle dépasse 1000 $\mu \mathrm{S} / \mathrm{cm}$ pour les échantillons $\mathrm{T} 1, \mathrm{R}$ et $\mathrm{P} 1$. Cette conductivité provoque une augmentation dans le taux de la salinité des eaux. La forte conductivité des eaux, peut être due soit à la nature des couches géologiques de la nappe (présence des grés et des calcaires), soit à la présence d'éléments minéraux indésirables dans les eaux et résultant d'une pollution exogène.

Les chlorures existent dans la quasitotalité des eaux à des concentrations très variables. Nous observons une forte concentration en chlorures dépassant les normes de l'OMS pour le forage T1, les autres échantillons ont une concentration en chlorures moins de $100 \mathrm{mg} / \mathrm{L}$ sauf pour les échantillons $\mathrm{R}, \mathrm{P} 1$ et $\mathrm{P} 3$ respectivement 251 $\mathrm{mg} / \mathrm{L}, 107,21 \mathrm{mg} / \mathrm{L}$ et $190,52 \mathrm{mg} / \mathrm{L}$. Ces deux éléments provoquent un goût désagréable dans nos eaux de consommation et la rendent corrosive vis-à-vis des conduites.

\section{Fluor}

Le fluor est présent dans les dattes, les aliments et le thé, il peut s'accumuler et présenter un danger pour la santé des populations et en particulier la fluorose sachant que la norme acceptable est de 0,60 à 0,80 mg/L (Achour et Youcef, 2001).

\section{Composés azotés}

Les ions ammonium $\left(\mathrm{NH}_{4}{ }^{+}\right)$subissent une nitration par action des bactéries nitrifiantes. L'azote nitreux $\left(\mathrm{NO}_{2}{ }^{-}\right)$provient d'une oxydation incomplète de l'azote ammoniacal ou par une réduction de nitrates par dénitrification. Les nitrites sont instables et sont rapidement transformés en nitrates. L'azote nitrique $\left(\mathrm{NO}_{3}{ }^{-}\right)$est produit par nitrification de l'azote ammoniacal.

Les nitrates peuvent être à l'origine de la formation de nitrites et de nitrosamines, responsables de deux phénomènes potentiellement pathologiques: la méthémoglobinémie et un risque de cancer. La présence des nitrates en concentration excessive peut provoquer un problème de santé publique important tel que la méthémoglobinémie, surtout pour les enfants de moins de 6 mois et les femmes enceintes ou nourrices au niveau des eaux d'alimentation de la ville de Tijikja la 
concentration maximale en nitrate est à 43,5 $\mathrm{mg} / \mathrm{L}$ au niveau du P3, elle est environ de 37 $\mathrm{mg} / \mathrm{L}$ au niveau de $\mathrm{T} 1$ et $\mathrm{P} 1$, ces concentrations sont inférieurs à la norme de l'OMS (50 mg/L). Pour les points TKF1, TKF2, FATTM et PT une faible concentration en nitrate ne dépassant pas $5 \mathrm{mg} / \mathrm{L}$ a été observée.

Les nitrites sont de puissants oxydants qui ont la capacité de transformer l'hémoglobine en méthémoglobine, rendant le sang incapable de transporter l'oxygène jusqu'aux tissus. De tels effets ont été observés chez de nombreuses espèces animales. Les nitrites sont toxiques pour l'organisme humain, sa présence en quantité importante dégrade la qualité de l'eau. L'ammonium étant toxique pour l'organisme humain, la présence en quantité importante dégrade la qualité de l'eau et un élément indicateur de la pollution. Les ions ammoniums non pas été détectés pour tous les échantillons.

\section{Métaux lourds}

L'analyse des métaux lourds des forages T1, TKF1, TKF2, FATTM, R et PT de Tijikja montre l'absence du fer et du cuivre, par contre les métaux tels que le chrome, le zinc, le cadmium et le plomb ont été détectés à des concentrations variables restant toujours loin des normes de l'OMS pour ces métaux.

Les éléments des traces métalliques (ETM) enregistrés montrent que leur origine ne pourrait être que géologiques et naturelle. Dans la mesure où les forages sont loin de la ville et qu'il n'a pas d'activité agricole, pastorale et industrielle

Enfin, si on compare les résultats des paramètres étudiés par rapport aux normes de qualité multi usages selon Bontoux (1983), on constate que ces eaux se situent entre la $2^{\text {eme }}$ et la $3^{\text {eme }}$ classe c'est-à-dire Bonne à passable.

\section{Conclusion}

Les différentes analyses effectuées sur les échantillons de l'eau prélevée au niveau de Tijikja ont permis de dresser un portrait de la qualité physico-chimique de ces eaux. Une comparaison des teneurs des principaux éléments mesurés dans les eaux souterraines de cette ville pour les différents points d'échantillonnage, indique une différence dans la concentration entre ces différents points de mesure. Cette différence est caractérisée par des valeurs élevées au niveau du forage $\mathrm{T} 1$, robinet $\mathrm{R}$, les puits $\mathrm{P} 1$ et $\mathrm{P} 3$.

En conséquence, nous pouvons dire que les eaux du forage $\mathrm{T} 1$ de la ville de Tijikja nécessitent un traitement physico-chimique avec des procédés d'adoucissement visant à diminuer la minéralisation avant qu'elles ne soient distribuées aux usagers.

\section{REMERCIEMENTS}

Nos vifs remerciements à tous les ingénieurs du Département de toxicologie et d'hydrologie d'hygiène environnemental et industriel de l'Institut National d'Hygiène de Rabat (Maroc), et à tous les ingénieurs et techniciens du Laboratoire de contrôle de la qualité des eaux de l'Institut National de Recherches en Santé Publique de Nouakchott (Mauritanie).

\section{REFERENCES}

Achour S, Youcef L. 2001. Excès des fluorures dans les eaux du Sahara septentrional oriental et possibilité de traitement. L'Eau, l'Industrie, les Nuisances, 247 : 47-54.

Bricha S, Ounine K, Oulkheir S, EL Haloui N, Attarassi B. 2007. Etude de la qualité physico-chimique et bactériologiques de la nappe phréatique M'nasra (Maroc), Afrique Science 3 (3) : 391-404.

Bontoux J. 1983. Introduction à l'étude des eaux douces, eaux naturelles, eaux usées, eaux de boisson, Partie III. La Tribune $d u$ Cebedeau, Liège, 36 : 241- 257. 
Centre Regional Agrhymet. 2005. Mensuel Agrhymet, $\mathrm{N}^{\circ}$ : M-06/ 05, Aout 2005.

Derwich D, Benabidat L, Zian A, Sadki O, Belghithy D. 2010. Caractérisation physico-chimique des eaux de la nappe alluviale du haut Sebou en aval de la confluence avec oued Fes. Larhyss/Journal, 8 : 101-112.

Guergazi S, Achour S. 2005. Caractéristique physico-chimiques des eaux d'alimentation de la ville de Biskra, pratique de chloration. Larhyss/Journal, 4 : 119-127.

Organisation Mondiale de Santé, 2006. Les lignes directrices de l'OMS en ce qui concerne la qualité de l'eau potable. www.lenntech.fr/applications/potable/nor mes/normes-oms-eau-potable.htm

Rodier J. 1996. L'Analyse de l'Eau : Eaux Naturelles, Eaux Résiduaires et Eaux de

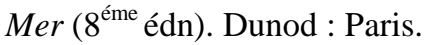

Tardat H, Beaudry J. 1984. Chimie des Eaux. Ed Le Griffon d'Argile INC : Québec.

World Health Organization. 1996. Surveillance of Drinking Water Quality. World Health Organization: Geneva.

www.gls.fr, 2005. Les analyses physicochimiques d'une eau. Memotec, $\mathrm{n}^{\circ} 32$. 